\title{
Note sur les populations relictuelles de Betula pendula Roth subsp. fontqueri (Rothm.) G. Moreno \& Peinado dans le Rif Centro-Occidental (Maroc).
}

\section{Hassan Ennouni \& Mohammed Ater}

Laboratoire Botanique Appliquée, Equipe Bio-Agrodiversité, Département de Biologie, Faculté des Sciences, Université Abdelmalek-Essaâdi, BP 2062, 93030, Tétouan, Maroc.

\author{
Correspondencia \\ M. Ater \\ e-mail: mohammed.ater@gmail.com \\ Recibido: 28 septiembre 2018 \\ Aceptado: 12 septiembre 2019 \\ Publicado on-line: 2 octubre 2019 \\ Editado por: T. Navarro
}

\begin{abstract}
Note on the relict populations of Betula pendula Roth subsp. fontqueri (Rothm.) G. Moreno \& Peinado in the Western Central Rif (Morocco)
\end{abstract}

Mots-clés: Betula pendula, relicte, phytoécologie, Liste Rouge régionale, Maroc.

Key words: Betula, pendula, relict, plant ecology, regional Red List, Morocco
Le Rif est reconnu comme un important hot spot de la biodiversité méditerranéenne et une zone refuge comportant des espèces relictes d'origine tertiaire (Quézel \& Médail 2003). Parmi ces relictes, on compte plusieurs espèces holarctiques et eurosibériennes en limite d'aire et dont l'isolement des populations a favorisé un important endémisme, souvent de niveau infraspécifique (Quézel 2000). Betula pendula Roth subsp. fontqueri (Rothm.) G. Moreno \& Peinado est un exemple de taxon relicte d'origine eurosibérienne considéré comme endémique ibéromarocain (Fennane et Ibn Tatou1998). II s'agit d'un taxon rare en danger d'extinction (Fennane 2017) et dont l'aire de répartition nord-africaine est limitée au Rif Centro-occidental. Pourtant, les données relatives à la localisation et la description des populations rifaines de cette espèce sont rares et anciennes, d'où l'intérêt de cette note présentant la localisation actuelle de ces populations et leur état de conservation.

\section{Populations de Betula pendula Roth en limite d'aire}

Betula pendula Roth est un arbre caducifolié de la famille des Bétulacées. C'est une plante monoïque, anémophile présentant un cytotype diploïde $(2 n=28)$ (Fischer et al. Jonsell 2000). Betula pendula est largement répandu en Europe et en Asie Mineure, y compris dans l'est de la Turquie, le nord de l'Irak et le nord de l'Iran (Atkinson 1992 ; Krussmann 1984). II est représenté en limite d'aire que ce soit en Asie ou en Europe par des populations isolées à faible densité. En Asie, on peut citer l'exemple de l'Iran (Yousefzadeh et al.
2016) où il est inscrit sur la liste rouge des plantes d'Iran en tant qu'espèce en voie de disparition (Jalili \& Jamzad 1999). De même, en Europe, on peut citer l'Espagne où les populations andalouses de l'espèce sont aussi inscrites sur la liste rouge (Blanca et al. 1999; Cabezudo et al. 2005 et Moreno 2008). Dans la péninsule ibérique et au nord du Maroc (Rif Centro-Occidental) l'espèce est représentée par une sous espèce endémique ibéromarocaine : Betula pendula Roth subsp. fontqueri. Bien qu'en Andalousie on parle de deux variétés au sein de cette sous-espèce (Moreno \& Peinado 1990), dans le nord du Maroc on ne fait pas cette distinction (Fennane et Ibn Tattou 2005 ; Valdés et al. 2002).

\section{Citations et observations de Betula pendula Roth au Maroc}

Dans la littérature botanique au Maroc, ce taxon a été cité sous différentes nomenclatures synonymes dont le basionyme est Betula fontqueri Rothm., in Bol. Soc. Brot., II, 14: 149. 1940. Dans le Catalogue des plantes du Maroc (Jahandiez \& Maire. 1932), l'Atlas du Maroc (Metro 1958) et dans la flore de l'Afrique du nord (Maire 1961), on cite Betula alba L. Des phytosociologues ont utilisé plutôt Betula celtiberica comme nom et ont même décrit une association spécifique au bouleau dans le Rif (Barbéro et al. 1981), Primulo acaulis Betuletum celtibericae.

Le premier échantillon d'herbier connu de ce taxon est déposé à l'herbier de l'Institut Scientifique de Rabat (RAB 029696). II a été récolté en 1927 par P. Font Quer dans la région de 
Kétama. Pour les données anciennes relatives à la localisation, description des populations et relevés de végétation, on peut citer les comptes rendus de l'excursion botanique au Maroc de 1965 publiés par Sauvage en 1971 et qui cite une seule localité à Bouleau située entre Telat-Ketama et Ketama et les 4 relevés de Barbéro et al. (1981) effectués dans la région de Ketama et le Jbel-Tidighine.

\section{Prospections et enquêtes de localisation}

Une exploration exhaustive du territoire des cédraies du Rif Centro-occidental a été effectuée au cours de l'année 2015. Elle s'est basée dans un premier temps sur des enquêtes auprès du personnel technique forestier et des populations locales. Ensuite, elle a été affinée par un protocole d'arpentage terrestre. Ce travail a permis d'identifier trois sites (Kétama, Dehdouh et Tidighine) où le bouleau est encore présent et d'en inventorier les individus (Fig.1).

Des relevés phytoécologiques ont été réalisés en 2016 et 2017 dans les sites où le bouleau a été localisé. Les principales caractéristiques des stations où les relevés ont été réalisés sont résumées dans le tableau 1 . La nomenclature adoptée pour les espèces identifiées est celle de la
Flore du Maroc (Fennane et al. 1999, 2007, 2014) et le catalogue des plantes vasculaires du nord du Maroc (Valdés et al. 2002).

\section{Situation actuelle des populations à bouleau dans le Rif}

La mise à jour des données sur le Bouleau a permis de constater une réduction drastique des sites occupés par cette essence par rapport aux données des prospections botaniques effectuées en 1978 (Barbéro et al. 1981). En effet, elle aurait disparue dans deux sites : Bab-bessen (ou BabEssam), à l'extrémité est du Rif Occidental et d'Imasinenn qui relève du Rif central, au pied de Jbel-Tidighine. Par contre, nos prospections ont montré deux nouveaux sites : Dehdouh et Tidighine. Un seul site persisterait alors des anciennes citations, c'est celui de la source d'Aachouch à Kétama. Dans tous les cas, les sites sont réduits à des tâches de quelques dizaines de mètres carrés. Cette situation ne semble pas récente, mais au contraire, relativement ancienne si on se réfère à Barbéro et al. (1981). D'autre part, l'effectif des populations est très faible ; l'effectif le plus important est de seulement 11 individus observés dans le site de Kétama. Toutefois, la présence d'individus

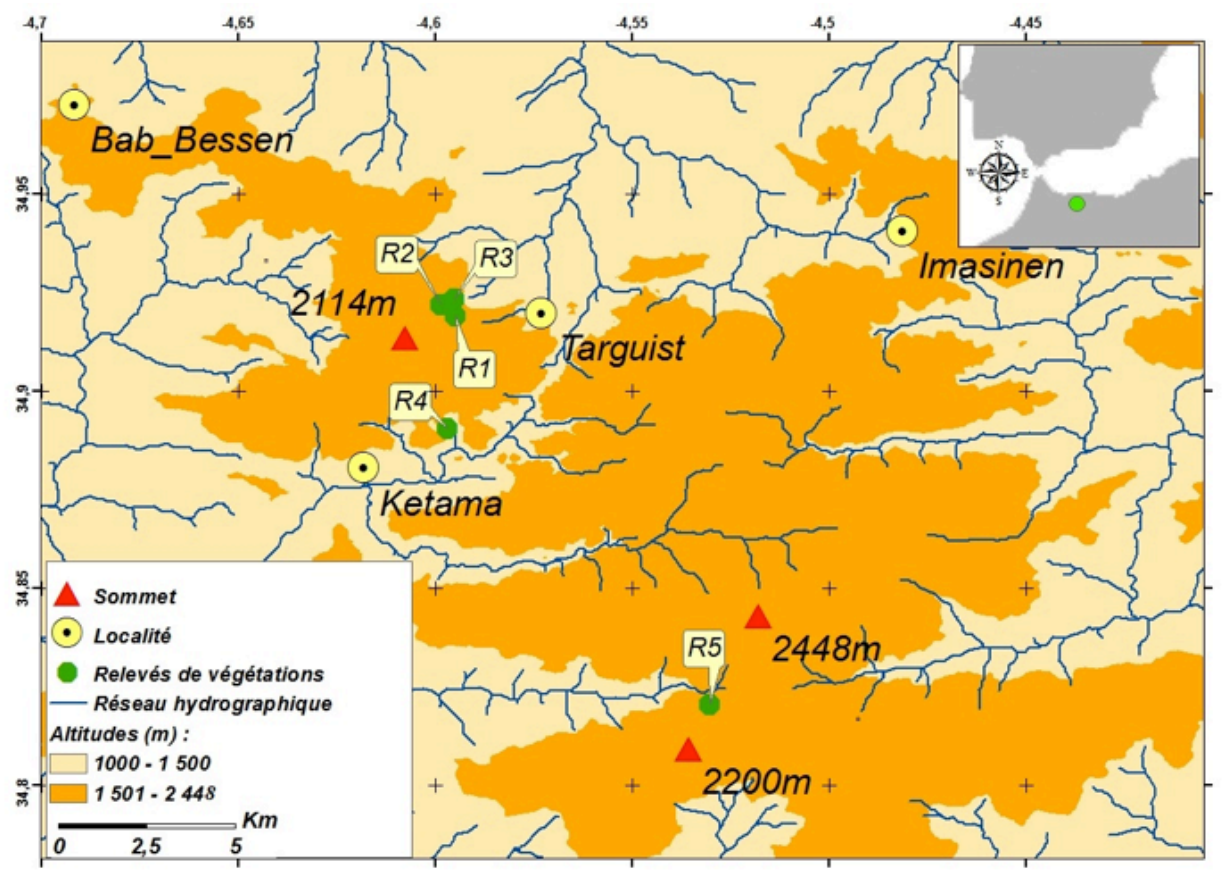

Figure 1 : Carte de localisation des sites à Betula pendula et des relevés de végétation (R1, R2, R3, R4 et R5) dans le Rif centro-occidental. Carte réalisée avec le logiciel Arc-Gis10.3 sur un fond illustrant l'altitude et le réseau hydrographique. En haut de la figure, localisation de la zone d'étude dans le nord du Maroc.

Figure 1: Site map of Betula pendula and vegetation surveys (R1, R2, R3, R4 and R5) in the Central West Rif. Map made with the software Arc-Gis10.3 on a background illustrating the altitude and the hydrographic network. At the top of the figure, location of the study area in northern Morocco. 


\begin{tabular}{|c|c|c|c|c|c|}
\hline Relevés & $\mathbf{R 1}$ & $\mathbf{R 2}$ & R3 & R4 & R5 \\
\hline Coordonnées WGS84 & $\begin{array}{r}- \\
4,59655 \mathrm{~W} \\
34,9216 \mathrm{~N}\end{array}$ & $\begin{array}{r}- \\
4,59877 \mathrm{~W} \\
34,9221 \mathrm{~N}\end{array}$ & $\begin{array}{c}-4,5951 \mathrm{~W} \\
34,9237 \mathrm{~N}\end{array}$ & $\begin{array}{r}- \\
4,595195 \mathrm{~W} \\
34,8881 \mathrm{~N}\end{array}$ & $\begin{array}{r}- \\
4,59498 \mathrm{~W} \\
34,9189 \mathrm{~N}\end{array}$ \\
\hline Altitude (m) & 1625 & 1675 & 1570 & 1454 & 1921 \\
\hline Hauteur (m) & 15 & 23 & 6 & 11 & 15 \\
\hline Substrat & $\begin{array}{r}\text { Schiste - } \\
\text { Grés }\end{array}$ & $\begin{array}{r}\text { Schiste - } \\
\text { Grés }\end{array}$ & $\begin{array}{r}\text { Schiste - } \\
\text { Grés }\end{array}$ & $\begin{array}{r}\text { Schiste - } \\
\text { Grés }\end{array}$ & $\begin{array}{r}\text { Schiste } \\
\text { - Grés } \\
\text { Calcaire }\end{array}$ \\
\hline Pente \% & 20 & 15 & 10 & 15 & 25 \\
\hline Habitat & $\begin{array}{l}\text { Cours } \\
\text { d'eau }\end{array}$ & $\begin{array}{r}\text { Source } \\
+ \text { cours } \\
\text { d'eau }\end{array}$ & $\begin{array}{r}\text { Source } \\
+ \text { cours } \\
\text { d'eau }\end{array}$ & $\begin{array}{c}\text { Source + } \\
\text { cours d'eau }\end{array}$ & $\begin{array}{l}\text { Cours } \\
\text { d'eau }\end{array}$ \\
\hline Formation végétale & Cédraie & Cédraie & Cédraie & $\begin{array}{l}\text { Matorral de } \\
\text { Chêne liège }\end{array}$ & Cédraie \\
\hline Couvert forestier (\%) & 100 & 100 & 95 & 40 & 100 \\
\hline Statut du terrain & Domanial & Domanial & Domanial & Privé & Domanial \\
\hline Localité & Dehdouh & Dehdouh & Dehdouh & Aachouch & $\begin{array}{r}\text { Jbel- } \\
\text { Tidighine }\end{array}$ \\
\hline & \multicolumn{5}{|c|}{ Indices de Braun-Blanquet } \\
\hline \multicolumn{6}{|l|}{ Cortège floristique } \\
\hline Betula pendula subsp. fontqueri & + & 1.1 & + & 2.2 & + \\
\hline Primula veris var. acaulis $\mathrm{L}$. & 2.2 & 3.4 & 1,1 & 1.1 & 2.2 \\
\hline Pteridium aquilinum (L.) Kuhn in Kersten & 2.2 & 3.3 & 2.2 & 1.1 & + \\
\hline Prunus Iusitanica L. & 2.2 & 2.2 & & 2.2 & 2.2 \\
\hline Cedrus atlantica (Endl.) Carrière & 1.1 & 5.5 & 3.3 & & 5.5 \\
\hline Geranium malviflorum Boiss. \& Reut. & + & 1.1 & & - & 1.1 \\
\hline Frangula alnus Mill. subsp. alnus & 1.1 & & & + & 1.1 \\
\hline Salix atrocinerea Brot. & 1.1 & & & 1.1 & 1.1 \\
\hline Aquilegia vulgaris $\mathrm{L}$. & + & & & 0 & + \\
\hline Bunium alpinum Wadst \& Kit. subsp. atlanticum Maire & + & & & - & + \\
\hline Vicia cedretorum Font Quer & & 1.1 & + & & + \\
\hline Digitalis purpurea var. mauritanica Emberger \& Maire & & + & 1.1 & & + \\
\hline Ilex aquifolium $\mathrm{L}$. & & 1.1 & & 2.2 & 3.3 \\
\hline Brachypodium sylvaticum (Huds.) P. Beauv. subsp. sylvaticum. & & 1.1 & 1.1 & & 1.1 \\
\hline Stellaria media (L.) Vill. & 1.1 & 1.1 & & 1.1 & \\
\hline Polystichum setiferum (Forsk.) Woyn & 1.1 & & & 1.1 & 2.2 \\
\hline Athyrium filix-femina (L.) Roth & & 1.1 & & 2.2 & \\
\hline Geum silvaticum Pourr. & & 1.1 & & & 1.1 \\
\hline Daphne laureola L. subsp. latifolia Coss. Rivas-Martinez & 2.2 & + & & & \\
\hline Rubus ulmifolius Schott & & 1.1 & & 3.3 & \\
\hline Tamus communis $\mathrm{L}$. & & & + & + & \\
\hline Viola reichenbachiana Jord. & & 2.2 & & & 1.1 \\
\hline Sanicula europaea L. & & 2.2 & & & 1.1 \\
\hline Potentilla micrantha Ramond & + & + & & & \\
\hline Blechnum spicant (L.) Roth & & & 2.3 & 2.2 & \\
\hline
\end{tabular}




\begin{tabular}{|c|c|c|c|c|c|}
\hline Relevés & $\mathbf{R 1}$ & $\mathbf{R 2}$ & R3 & R4 & R5 \\
\hline Quercus rotundifolia Lam. & & + & & + & \\
\hline Sedum forsterianum Smith. & & 2.2 & & & 2.2 \\
\hline Prunella vulgaris $\mathrm{L}$. & & + & & 1.1 & \\
\hline Cardamine hirsuta L. & & + & 1.1 & & \\
\hline Galium ellipticum Willd. & & 1.1 & 1.1 & & \\
\hline Scrophularia auriculata Loefl. (= S. aquaticaL. var. laxa Maire) & & + & + & & \\
\hline Lamium flexuosum Ten. & & + & & & + \\
\hline Taxus baccata L. & & & & & + \\
\hline Osmunda regalis $\mathrm{L}$. & & & & 2.3 & \\
\hline Dryopteris filix-mas (L.) Schott & & & & & + \\
\hline Luzula forsteri DC. & & & & & 1.1 \\
\hline Satureja baborensis (Batt.) Briq. & & + & & & \\
\hline Acer opalus subsp. granatense (Boiss.) Font Quer \& Rothm. & & & & & 1.1 \\
\hline Sorbus aria (L.) Crantz subsp. meridionalis (Guss.) Kerner & & & & & + \\
\hline Ranunculus ficaria L. subsp. ficariiformis Rouy \& Fouc. & & 1.1 & & & \\
\hline Clematis vitalba L. & & & & 1.1 & \\
\hline Ranunculus macrophyllus Desf. & & & & 1.1 & \\
\hline Quercus canariensis Willd. & & 1.1 & & & \\
\hline Hedera maroccana Mc Allister (= Hedera helix L.) & & & & 2.2 & \\
\hline Cystopteris fragilis (L.) Bernh. & & & & & 1.1 \\
\hline Rubia peregrina L. & & & & 1.1 & \\
\hline Carex paniculata $\mathrm{L}$. & & & & 2.2 & \\
\hline Lonicera arborea Boiss. & & & & + & \\
\hline Scirpoides holoschoenus (L.) Soják & & & & 2.2 & \\
\hline Agropyron marginata $\mathrm{H}$. Lindb. & & & 1.1 & & \\
\hline Satureja vulgaris (L.) Fritsch & & & 1.1 & & \\
\hline Smilax aspera L. subsp. mauritanica (Poiret) Malag. & & & & 1.1 & \\
\hline Stellaria holostea L. & & & 1.1 & & \\
\hline
\end{tabular}

Tableau 1 : Tableau des relevés floristiques des sites à Betula pendula dans le Rif.

Table 1 : Synthetic table of floristic releves of the sites at Betula pendula the Rif.

jeunes montre l'existence d'un intéressant potentiel de régénération chez cette espèce.

Statut de Betula pendula Roth subsp. fontqueri (Rothm.) sur la liste rouge de la flore vasculaire au Maroc

La mise à jour des données concernant le bouleau et sa répartition dans le du Rif Centrooccidental permet de dresser un constat alarmant sur la pérennité des peuplements. La confrontation des données obtenues avec les critères de I'UICN (2012) pour la catégorie « en danger critique $(\mathrm{CR})$ ", montre que ce taxon remplit à l'échelle régionale au moins deux critères (A et $\mathbf{D})$ permettant de le considérer comme confronté à un risque élevé d'extinction. Ainsi, en ce qui concerne le critère $\mathbf{A}$ relatif à la répartition géographique, la zone d'occurrence est nettement inférieur au seuil de $100 \mathrm{Km}^{2}$, car elle ne dépasserait guère $12 \mathrm{Km}^{2}$ avec un déclin manifeste du nombre de localités et du nombre d'individus. Nous avons également, constaté que cette espèce a disparu dans certaines localités où elle a été observée précédemment. D'autre part, en ce qui concerne 
le critère $\mathbf{D}$, nous avons recensé un effectif faible ne dépassant pas 19 pieds et qui est largement inférieur au seuil de 50 individus préconisé pour ce critère pour la catégorie UICN (CR). Ainsi, on est en droit de penser que le statut de Betula pendula Roth subsp. fontqueri (Rothm.) sur la liste rouge régionale (Fennane 2017) doit être révisé. En effet, il serait opportun de remplacer le statut actuel d'espèce en danger (EN) par la catégorie taxon en danger critique d'extinction (CR). Les habitats occupés par cette espèce sont fortement menacés par l'anthropisation du milieu (coupe, captage d'eau, défrichement...). Des mesures urgentes doivent être prises aussi bien in situ pour conserver les derniers peuplements existants, qu'en ex situ (semences et boutures) pour conserver ce patrimoine génétique.

\section{Remerciements}

Nous tenons à remercier les responsables des Directions Régionales des Eaux et Forêts et de la Lutte Contre la Désertification du Rif et du Nord-Est pour les moyens humains et matériels mis à notre disposition pour faciliter les investigations et enquêtes sur le terrain.

\section{Bibliographie}

Atkinson, M. (1992). Betula pendula Roth (B verrucosa Ehrh.) and B. pubescens Ehrh. Journal of Ecology, 80(4), 837-870.

Barbéro, M., Quézel, P., \& Rivas-Martínez, S. (1981). Contribution à l'étude des groupements forestiers et préforestiers du Maroc. Phytocoenologia, 9(3), 311412.

Blanca, G., Cabezudo, B., Herrera, C. M., Molero Mesa, J., Muñoz, J., \& Valdés, B. (1999). Libro Rojo de la Flora Silvestre Amenazada de Andalucía. Tomo I: Especies en Peligro de Extinción. Consejeria de Medio Ambiente. Junta de Andalucia.

Cabezudo, B., Tlavera, S., Blanca G., Salazar C., Cueto, M., Valdés, B., Hernandés Bermejo, J.E., Herera, C. M., Rodriguez Hiraldo., C., \& Navas., D. (2005). Lista roja de la flora vascular de Andalucia. Consejeria de Medio Ambiente. Junta de Andalucìa.

Fennane, M., Ibn Tattou, M., Mathez, J., Ouyahya, A., \& EL Oualidi, J. (1999). Flore Pratique du Maroc, volume 1. Trav. Inst. Sci., sér. Bot. 36. Rabat.1-560 p.

Fennane, M., \& Ibn Tattou, M. (1998). Catalogue des plantes rares, menacées ou endémiques du Maroc. Bocconea 8. Palerme. 1-243 p.

Fennane, M., \& Ibn Tattou, M. (2005). Flore vasculaire du Maroc: Inventaire et Chorologie. Volume 1 Pteridophyta, Gymnospermae, Angiospermae p.p. Trav. Instit. Sci. Sér. Bot. 37. Rabat : 1- 489.

Fennane, M., Ibn Tattou, M., Mathez, J., Ouyahya, A., \& EL Oualidi, J. (2007). Flore pratique du Maroc. Volume 2. Trav. Inst. Sci., sér. Bot. 38. Rabat.1-652 p.
Fennane, M., Ibn Tattou, M., \& EL Oualidi, J. (2014). Flore pratique du Maroc. Volume 3. Trav. Inst. Sci., sér. Bot. 40. Rabat.1-793.

Fennane, M. (2017). Eléments pour un Livre rouge de la flore vasculaire du Maroc. Fasc. 4. Basellaceae Buxaceae. Edt. Tela-Botanica.

Fischer, A., Lindner, M., Abs, C., \& Lasch, P. (2002). Vegetation dynamics in Central European forest ecosystems (near-natural as well as managed) after storm events. Folia Geobotanica, 37(1), 17-32.

Jahandiez, E. \& Maire, R. (1932). Catalogue des plantes du Maroc (Spermatophytes et Ptéridophytes) Tome 2. Minerva. Alger.

Jalili, A. \& Jamzad, Z. (1999). Red Data Book of Iran:a preliminary survey of endemic, rare \& endangered plant species in Iran. Research Institute of Forests \& Rangelands. Tehran. Iran.

Jonsell, B. (2000). Flora Nordica Volume 1. Lycopodiaceae - Polygonaceae. The Bergius Foundation, The Royal Swedish Academy of Sciences. Stockholm. Sweden.

Krussmann, G. (1984). Manual of cultivated broadleaved trees and shrubs. Timber press. London.

Maire, R. (1961). Flore de l'Afrique du Nord. Volume VII. Editions Le Chevalier. Paris.

Metro, A. (1958). Forêts. Atlas du Maroc. Notices explicatives. Section VI Biogéographie forêts et ressources végétales. Rabat.

Moreno, G., \& Peinado, M. (1990). Betula L. pp. 38-43. in Castroviejo et al., eds., (1989-) Flora Ibérica. Plantas Vasculares de la Península Ibérica e Islas Baleares. Vol.II.: Platanaceae-Plumbaginaceae (partim). Real Jardín Botánico, CSIC. Madrid. 897 pp.

Moreno, J.C., coord. (2008). Lista Roja 2008 de la flora vascular española. Dirección General de Medio Natural y PolíticaForestal (Ministerio de Medio Ambiente, y Medio Rural y Marino, y Sociedad Española de Biología de la Conservaciónde Plantas), Madrid. 86 pp.

Quézel, P., (2000). Réflexions sur l'évolution de la flore et de la végétation au Maghreb méditerranéen. Ibis press. Paris

Quézel, P., \& Médail, F. (2003). Ecologie et biogéographie des forêts du bassin méditerranéen. Ed. Lavoisier. $571 \mathrm{pp}$

Rothmaler, W., \& de Carvalho Vasconcellos, J. (1940). Betula celtiberica Rothm. Bol. Soc. Brot., ser. 2, 14: 139-188.

Sauvage C., (1971). Excursion botanique au Maroc. Al Awamia n41: 105-210.

UICN, (2012). Lignes directrices pour l'application des Critères de la Liste rouge de I'UICN aux niveaux régional et national. Version 4.0. (janvier 2010). Gland, Suisse et Cambridge, Royaume-Uni, UICN. iv $+44 \mathrm{pp}$.

Valdés, B., Rejdali, M., Achhal El Kadmiri, A., Jury, S. L., \& Montserrat, J. M., (2002). Catalogue des plantes vasculaires du nord du Maroc. Volume I et II.: Consejo Superior de Investigaciones Científicas, Madrid.

Yousefzadeh, H., Colagar, A. H., \& Fallah, F. (2016). Populations of Betula pendula Roth : implications for conservation. Silva Fennica, 50(3), 1-12. 